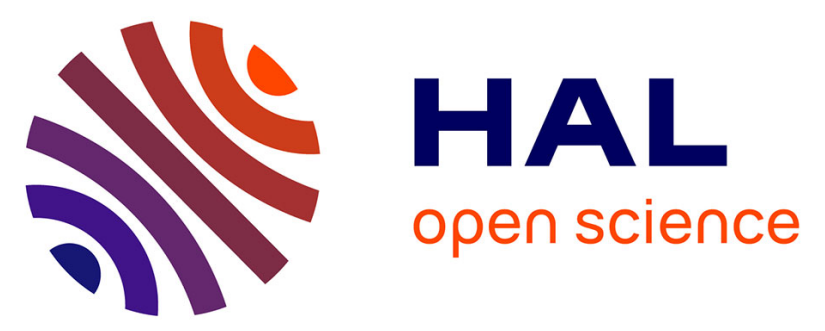

\title{
Réseaux et environnement : regards croisés sur les filières de gestion des Déchets d'Équipement Électriques et Électroniques à Toulouse et à Milan
} Jean-Baptiste Bahers, Isabella Capurso, Cédric Gossart

\section{- To cite this version:}

Jean-Baptiste Bahers, Isabella Capurso, Cédric Gossart. Réseaux et environnement : regards croisés sur les filières de gestion des Déchets d'Équipement Électriques et Électroniques à Toulouse et à Milan. Flux - Cahiers scientifiques internationaux Réseaux et territoires, 2015, 99, pp.32 - 46. 10.3917/flux.099.0032 . hal-01258015

\section{HAL Id: hal-01258015 https://hal.science/hal-01258015}

Submitted on 18 Jan 2016

HAL is a multi-disciplinary open access archive for the deposit and dissemination of scientific research documents, whether they are published or not. The documents may come from teaching and research institutions in France or abroad, or from public or private research centers.
L'archive ouverte pluridisciplinaire HAL, est destinée au dépôt et à la diffusion de documents scientifiques de niveau recherche, publiés ou non, émanant des établissements d'enseignement et de recherche français ou étrangers, des laboratoires publics ou privés. 


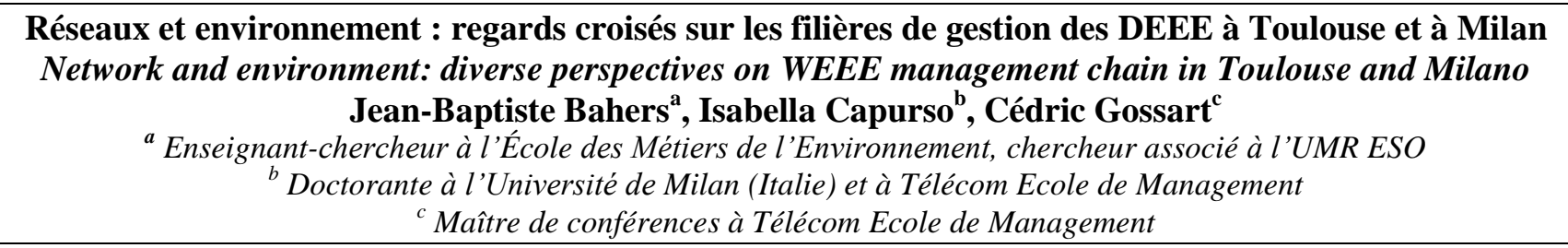

\title{
Résumé
}

Le principal objectif de cet article est d'étudier le cadre institutionnel d'une filière de gestion des déchets par une entrée sur les modalités de gouvernance des réseaux d'acteurs, afin de répondre à la question de recherche suivante : comment peut-on expliquer les différences d'adaptation des systèmes sociotechniques de gestion des DEEE (Déchets d'Équipements Électriques et Électroniques) italien et français à la mise en œuvre de la directive européenne DEEE ? Cette recherche aborde la construction locale de la filière de gestion en Italie et en France en réponse aux objectifs de territorialisation de la directive DEEE. Elle se focalise sur le management des DEEE à l'échelle urbaine, en menant une comparaison entre les villes de Milan et de Toulouse.

Le cadre empirique et théorique est construit pour évaluer le fonctionnement du modèle «Clearing house » au travers des relations entre les acteurs impliqués dans la filière: les citoyens-consommateurs, les collectivités territoriales, le système de distribution, les producteurs d'équipements électriques et électroniques, les éco-organismes, le secteur de l'économie sociale et solidaire (ESS) et les opérateurs de traitement. Le concept théorique des régimes sociotechniques est mobilisé pour étudier cette configuration particulière de filière résultant des interrelations entre les parties prenantes à plusieurs niveaux : 1) Dimension normative macro, c'est-à-dire la Directive Européenne relative aux DEEE et sa transposition en France et en Italie ; 2) Dimension méso : la mise en œuvre du dispositif réglementaire des DEEE à l'échelle urbaine dans les deux contextes (elle implique un alignement des facteurs législatifs, logistiques, économiques et socio-culturels, que nous appellerons des «régimes sociotechniques »); 3) Dimension micro : c'est à ce niveau que se produisent les changements technologiques majeurs au sein de "niches technologiques ».

Mots clés : DEEE, réseaux d'acteurs, régime sociotechnique, échelle urbaine.

\begin{abstract}
The general aim of this research is to explain the differences of adaptation of the French and Italian e-waste management regimes to the European WEEE directive. It draw insights on the concept of institutional chain by examining waste governance at different levels. In particular, this article focuses on e-waste management at urban scale, carrying out a comparative case-study between Milan and Toulouse. In this, the analysis reconstructs in Italy and France the supply chain that has been built to pursue the objectives set by the European WEEE Directive, and then it gets to the heart of its application at urban scale.

The empirical and theoretical interests driving the analysis is to evaluate the overall (dis)functioning of the "Clearing House" model through the relationships between involved actors: citizens-consumers, local authorities, system of provision, hi-tech producers (via eco-organisms), third sector (social economy) and treatment operators. In this approach, the concepts of socio-technical regime and transition management have been deemed useful tools to address the issue at stake, since it is believed that the final configuration of the supply chain is the result of the reciprocal interaction between the following factors: 1) A macro normative framework represented by the WEEE Directive and its implementation in France and Italy; 2) A meso dimension, which corresponds to the local implementation on urban scale of the latter regulatory framework (this enforcement implies a general realignment of several factors: normative, logistical, economical and socio-cultural, which will be referred to as "socio-technical regime"); 3) A micro level, which is represented by technological niches where radical innovations occur.
\end{abstract}

Key words: e-waste, stakeholder network, socio-technical regime, urban scale. 


\section{Introduction}

Les déchets d'équipements électriques et électroniques (DEEE) forment une vaste famille d'appareils qui cristallisent de nombreuses inquiétudes en termes d'impacts environnementaux et sociaux (Kuehr et al., 2003 ; Huisman, 2008 ; Flipo et al., 2009; Breuil et al., 2009, ÉcoInfo, 2012), et cela à l'échelle de toute la planète (Gossart, 2009). En particulier, dans les pays de l'Union Européenne une transition importante se construit concernant la gestion des DEEE selon une Directive européenne récente et contraignante en termes de collecte et traitement des DEEE. Ainsi, les politiques de gestion des DEEE représentent un objet d'étude très riche qui permet d'explorer comment une réglementation européenne se transpose dans les Etats, et comment cette transposition s'adapte aux spécifiés locales (tels que culturelles, sociales, économiques, politiques et infrastructurelles). Dans cet article, nous proposons donc d'analyser la territorialisation des politiques de gestion des DEEE à Milan et à Toulouse, à partir de l'approche théorique des régimes sociotechniques (Geels et al. 2007). Cette grille de lecture permet de valoriser l'aspect multiscalaire des politiques de transition écologique (Geels, 2002), en mettant en lumière la relation mutuelle entre ces différents niveaux impliqués dans le changement (macro, meso, micro). En particulier, cette proposition postule que la Directive Européenne relative à la gestion des DEEE, qui impose aux pays membres des règles uniformes et des cibles communes, représente un élément macro de rupture par rapport aux systèmes antérieurs de gestion des DEEE en France et en Italie. Une interrogation découle de cette hypothèse : comment ce «choc» (selon le terme de Geels) engendre-t-il une réorganisation interne des régimes normatifs, logistiques, socio-culturels et économiques liés à la gestion des DEEE aux deux échelles nationale et urbaine ? Ces derniers éléments forment un ensemble complexe et dense qui représente la dimension méso de notre analyse. Enfin, la contingence d'autres modifications des régimes sociotechniques est étudiée à partir des niches technologiques, où se produisent les innovations radicales porteuses de changements sociotechniques substantiels.

Avant d'entrer dans les détails de l'analyse des régimes sociotechniques, il est nécessaire de présenter l'approche théorique qui conduit cette recherche (Section 2). Ensuite, nous pourrons aborder la gestion des DEEE en Italie et en France, car la Directive s'impose tout d'abord dans le cadre réglementaire national (Section 3). Enfin, l'étude de cas comparative sera décrite selon les trois niveaux macro, méso, micro (Section 4).

\section{La territorialisation des politiques de gestion des DEEE : Transition écologique et système sociotechnique}

Étudier les mécanismes de mise en œuvre de la Directive Européenne des DEEE à l'échelle urbaine implique d'interroger un phénomène complexe et multiscalaire qui s'inscrit dans la réflexion de l'approche de la transition. Pour Loorbach et al. (2011), l'approche de la transition permet de comprendre et d'orienter notre société vers le développement durable en jouant à la fois sur la vitesse de transition et sur sa direction (la soutenabilité des sociétés humaines). Elle permet pour y parvenir d'élaborer des scénarios soutenables et des guides de gouvernance des systèmes sociotechniques. Pour ces spécialistes néerlandais de la transition vers un développement durable :

«L'approche de la transition prend en compte les caractéristiques intrinsèques $\mathrm{du}$ système social (e.g. sa complexité, l'interdépendance des composantes du système, la présence d'acteurs et de réseaux multiples), et permet d'appréhender les changements fondamentaux dans la culture, les structures et pratiques sociétales comme autant de réponses à des problèmes récurrents. » (notre traduction)

Boulanger (2008) souligne également que cette approche unit « un corps de concepts et d'hypothèses pour l'analyse et l'explication du changement et de l'innovation sociotechniques » avec «un ensemble de principes et des méthodes pour la gouvernance de ce type de changement ». Pour Kemp \& Rotmans (2004), une transition implique le passage d'un équilibre dynamique initial à un nouvel équilibre dynamique (un régime), des développements rapides et lents résultant de nombreuses interactions, et des innovations dans d'importantes parties des sous-systèmes sociétaux. Cette approche multi-niveaux (multi-level perspective, MLP) conçoit les transitions comme des alignements entre dynamiques à l'œuvre à différents niveaux sociétaux (macro, meso, micro), et propose une typologie des transitions. Celles-ci diffèrent de part leur timing et la nature des interactions à l'œuvre à chaque niveau sociétal. La figure suivante propose une représentation graphique de cette approche en partant du niveau micro en bas (« Niche level») au niveau macro tout en haut («Landscape developments ») en passant par le régime sociotechnique du milieu. 
Figure 1 : Représentation de l'approche des systèmes sociotechniques

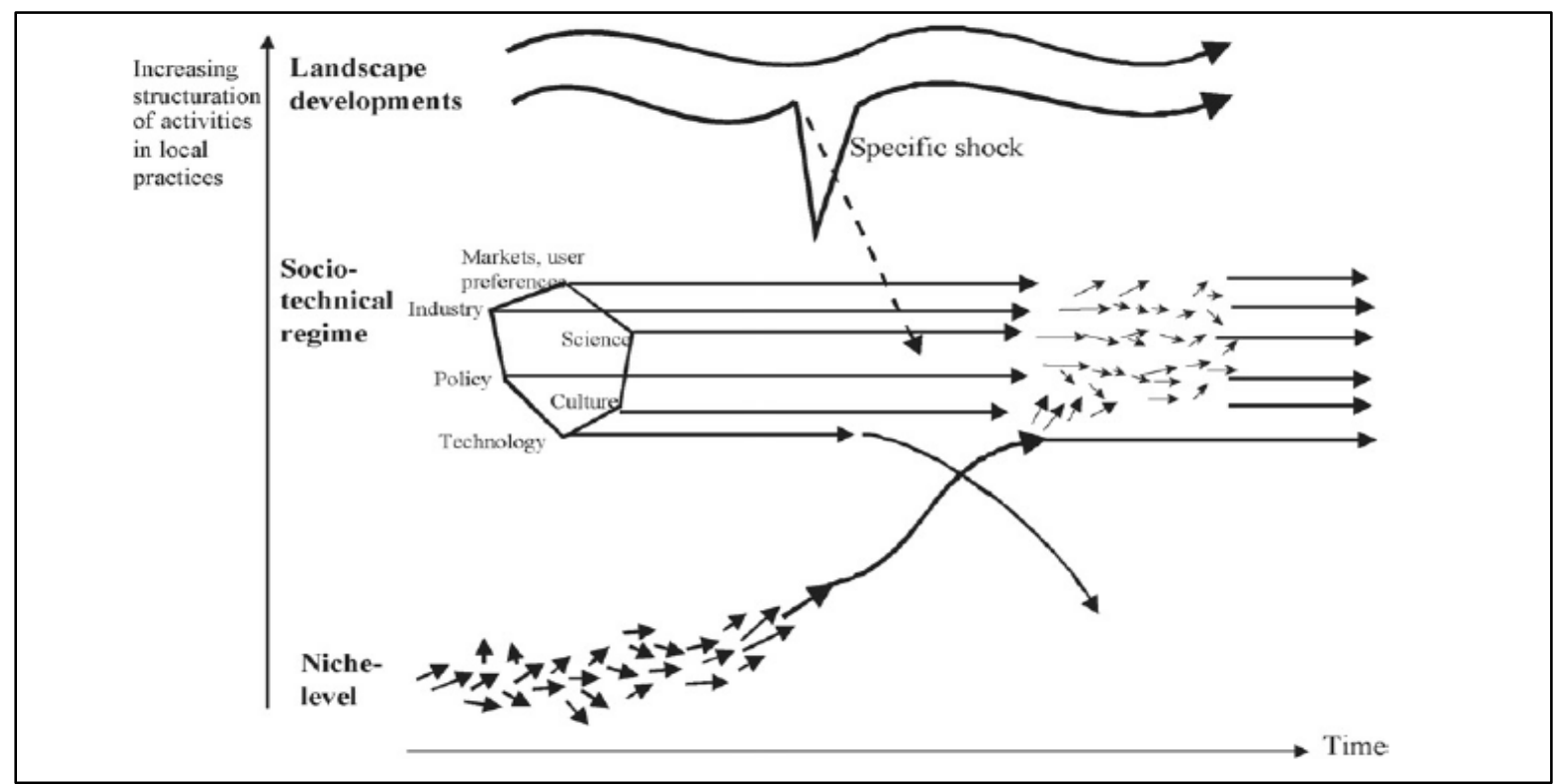

Source : Geels \& Schot 2007, p. 407.

Pour Boulanger (2008) :

« De façon générale, cette transition résulte de l'apparition de multiples changements qui se produisent simultanément à différents niveaux et dans différents secteurs de la société (la technologie, l'économie, les institutions, les comportements, la culture, l'écologie...) et qui se renforcent et s'amplifient mutuellement jusqu'à entraîner — en cas de transition réussie - une reconfiguration globale du système considéré. »

Les différents niveaux d'influence sont au nombre de trois, classés par ordre descendant de complexité sociétale (du macro au micro) :

- Le paysage sociotechnique (niveau macro) : le plus difficile à modifier, ce niveau constitue l'environnement exogène aux deux niveaux inférieurs, et comprend par exemple les grandes tendances macroéconomiques et politiques, les caractéristiques culturelles profondes, ou les modifications globales des écosystèmes. Notre recherche postule que la Directive Européenne DEEE représente un facteur de changement à ce niveau, un «choc extérieur » impliquant nécessairement des impacts sur les régimes sociotechniques sous-jacents.

- Le régime sociotechnique (niveau meso) : il est le niveau clé de l'approche de la transition car il assure la stabilité d'un régime sociotechnique donné, et c'est donc en le modifiant que l'on parviendra à pérenniser des solutions favorables au développement durable. Il regroupe les communautés de groupes sociaux ainsi que l'ensemble de leurs activités. Ce niveau d'analyse comprend également les règles et moyens d'interaction des acteurs. Notre recherche considère que la mise en œuvre à l'échelle urbaine de la Directive DEEE mobilise toute une série de facteurs (réglementaires, logistiques, économiques, socio-culturels) qui s'inscrivent parfaitement dans la définition des régimes sociotechniques. Ils sont donc interrogés par rapport à leurs relations réciproques, et dans leur ensemble en tant que phénomène historique complexe.

- Les niches technologiques (niveau micro) : Ce niveau d'analyse porte sur les innovations sociotechniques capables de modifier le régime en place. Difficilement identifiables avant qu'une transition ait lieu, elles sont qualifiées de «radicales » mais n'étaient avant d'émerger que des configurations sociotechniques peu performantes au regard du changement désiré. Elles agissent comme des chambres d'incubation qui protègent les initiatives porteuses d'un changement soutenable, et peuvent être portées par de petits réseaux d'acteurs très motivés et souvent marginaux. Dans notre cas, ces niches peuvent être représentées par des évolutions techniques dans les domaines de l'écoinnovation et écoconception. 
La complexité de la gestion des DEEE fait de ce problème sociétal un bon cas d'étude pour appliquer l'approche de la transition aux cas français et italien. Cette approche permet d'examiner l'évolution de ce réseau dans une perspective historique riche d'enseignements pour l'avenir.

\section{Interroger les divergences dans les filières de gestion du déchet électronique en Italie et en France}

\subsection{La territorialisation des dispositifs réglementaires}

La première Directive DEEE (2002/95/CE), et les applications qui ont suivi ${ }^{1}$, est transposée dans le droit interne en Italie par le Décret Lgs. 151/2005 et dans le droit français par le Décret 829/2005. Ces transpositions représentent la base juridique pour la gestion des DEEE dans les deux pays. Les décrets, conformément à la Directive Européenne, contiennent des dispositions relatives aux principes suivants :

1. La prévention et la réduction des DEEE en amont ;

2. La promotion de la réutilisation et du recyclage des DEEE;

3. La définition des rôles et responsabilités des acteurs impliqués dans la gestion des DEEE (producteurs, revendeurs, consommateurs, communes, tiers secteur, etc.) ;

4. La réduction des substances toxiques et dangereuses contenues dans les produits électroniques et les déchets dérivés.

La structure de gestion, permettant la mise en place de ces principes, est opérationnelle en Italie en novembre 2007 et en France ${ }^{2}$ à partir de novembre $2006^{3}$. Les deux pays ont adopté un modèle de gestion identique portant le nom de «clearing house », qui se définit comme :

«Un cadre national dans lequel les différents acteurs (producteurs des EEE, recycleurs et autres opérateurs de la gestion des déchets) sont impliqués dans la gestion des DEEE. C'est au gouvernement d'assurer la création et la mise à jour d'un registre des producteurs, de définir les mécanismes d'attribution des DEEE à collecter et traiter parmi les différents acteurs concernés, ainsi que de mettre en place et gérer un système de surveillance. Un organisme national de coordination de la filière des DEEE est ainsi créé pour garantir le bon fonctionnement de la filière selon des critères de coûts et d'optimisation du système. ${ }^{4}$

Dans ce cadre et conformément au principe de responsabilité élargie du producteur (REP), les producteurs assument la responsabilité financière de la gestion des déchets électroniques, en fonction des produits qu'ils ont mis sur le marché chaque année. Pour ce faire, les producteurs ont l'obligation en Italie et l'option en France de se regrouper sous la forme d'éco-organismes ${ }^{5}$. Dans ce cadre, les éco-organismes italiens sont constitués sous l'arbitrage d'un organe de coordination, le Centro di Coordinamento (CDC RAEE'), et ils agissent dans un contexte de libre concurrence. Par contre, en France ils sont libres de s'organiser sans l'aval de leur organe de coordination (l'OCAD3E $\mathrm{E}^{7}$ ), même s'ils agissent également dans un contexte de libre concurrence ${ }^{8}$.

\footnotetext{
${ }^{1}$ La Directive 2002/95/CE a été soumis à une série d'ajouts et changements, qui ont abouti à une autre version plus récente, qui est la 2012/19/UE, publiée dans le Journal officiel de l'UE Le 24 juillet 2012. Cette dernière Directive doit être transposée en Droit interne par les Pays Membres entre le 14 février 2014.

${ }^{2}$ Pour les D3E ménagers.

${ }^{3}$ La directive a obligé les États membres à transposer cette loi dans leurs législations nationales avant le 13 août 2004 et, d'ici au 13 août 2005. La France et l'Italie ont en commun d'avoir été rappelées à l'ordre pour ne pas l'avoir transposée dans les délais établis (Draetta et Centemeri, 2007).

${ }^{4}$ Traduit selon nous à partir de : «A national framework in which multiple partners (producers, recyclers, and waste organizations) can provide services. The government ensures that there is a register of producers and defines the allocation mechanisms, together with the reporting and monitoring systems. A central national coordination body determines the well proper functioning of the entire system, according to criteria of cost and service optimization. » (Adelphi et al., 2008, p. 26)

${ }^{5}$ Pour les DEEE qui dérivent des produits mis sur le marché avant décembre 2010. Après cette date, la Nouvelle Directive introduit l'option de la responsabilité individuelle dans la gestion.

${ }^{6}$ Rifiuti da Apparecchiature Elettriche ed Elettroniche, DEEE en italien.

${ }^{7}$ Organisme Coordonnateur Agréé pour les DEEE.

${ }^{8}$ Il y a une pluralité d'éco-organismes qui agissent dans un contexte de concurrence, mais sous la supervision et coordination d'un arbitre qui assure une répartition équitable des quotas de collecte parmi les éco-organismes, ainsi que la garantie du service sur tout le territoire national.
} 
Les éco-organismes sont donc créés par et pour les producteurs d'EEE, en vue d'assumer leur responsabilité de prise en charge financière et organisationnelle de la filière. Ils sont donc en dialogue permanent avec les interlocuteurs majeurs de la chaîne de gestion des DEEE : les communes et intercommunalités, la distribution, l'économie sociale et solidaire (ESS), et les opérateurs de traitement.

Dans les deux pays, les collectivités doivent organiser un système de reprise pour les DEEE ménagers, et mettre à la disposition des citoyens des points de collecte tout en menant des campagnes de communication pour les informer des enjeux et solutions mises à leur disposition. En Italie comme en France, la gestion des DEEE est financée par un système de contribution financière organisé par les producteurs ${ }^{9}$ (ainsi que par la taxe sur l'enlèvement des ordures ménagères comme pour tous les déchets ménagers). Lors de l'achat d'un équipement électrique, le consommateur paye une contribution financière pour la fin de vie des produits ou éco-contribution (visible fee en anglais : « contribution visible »). Les distributeurs et producteurs récupèrent cette éco-contribution, ce qui permet de financer les éco-organismes pour la collecte et le traitement des DEEE. Enfin, les consommateurs sont invités à apporter leurs déchets dans les points de collecte mis à disposition par les municipalités. Mais ce système de gestion n'est pas homogène au sein d'un même pays, car il dépend des contextes locaux (caractéristiques sociodémographiques du territoire, performance générale du tri sélectif, présence de points de collecte de proximité, DEEE collectés dans le passé, densité démographique, etc.).

Les consommateurs ont également la possibilité de laisser leurs appareils électroniques aux revendeurs de produits Hi-Tech lors de l'achat d'un nouveau produit équivalent. Ce mécanisme obligatoire (appelée reprise « 1 pour 1 ») est prévu par la directive Européenne DEEE: c'est au distributeur d'organiser le bon fonctionnement du service « 1 pour $1 »$ (vente en ligne comprise), en fournissant des espaces pour stocker les DEEE dans les magasins et des points de collecte où les ils seront regroupés vers les déchetteries municipales agréées ou vers des recycleurs (quand les distributeurs gèrent leurs DEEE de manière indépendante).

Ce bref résumé permet de reconstruire les modalités de relations financières et matérielles de la filière des DEEE basée sur le modèle du « Clearing house », il est synthétisé pour les DEEE ménagers dans la figure 2.

Figure 2 : le mécanisme du «Clearing house » pour les DEEE ménagers

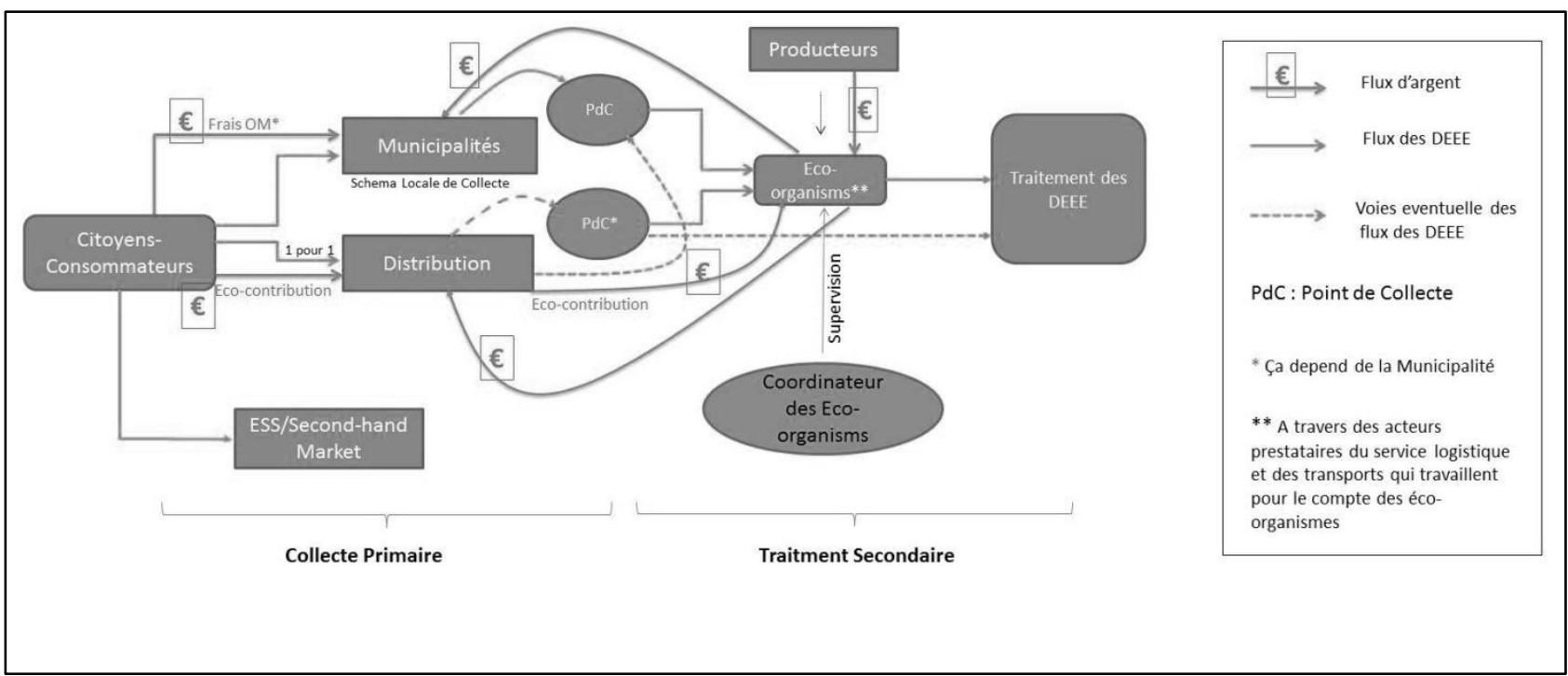

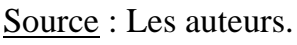

Avant de passer à l'analyse des relations entre acteurs du réseau DEEE en France et en Italie, il convient de souligner les principales divergences entre les deux pays.

\footnotetext{
${ }^{9}$ Selon la Nouvelle Directive DEEE (2012) en tant que « producteur » on entend également le fabricant et les importateurs des EEE.
} 


\subsection{Synthèse des principales divergences France / Italie}

Avant d'entamer les divergences quant aux flux de DEEE en Italie et en France, rappelons dans le tableau suivant sur les principales différences dans la territorialisation des dispositifs réglementaires.

Tableau 1 : Divergences des systèmes en Italie et en France

\begin{tabular}{|c|c|c|}
\hline & Italie & France \\
\hline $\begin{array}{l}\text { Profils des éco- } \\
\text { organismes }\end{array}$ & $\begin{array}{l}17 \text { éco-organismes aux profils divers et } \\
\text { variés }{ }^{10} \text {. }\end{array}$ & $\begin{array}{l}4 \text { sociétés à but non lucratif créées avec une } \\
\text { certaine cohérence sectorielle par des producteurs } \\
\text { membres des mêmes fédérations } \\
\text { professionnelles. }\end{array}$ \\
\hline $\begin{array}{l}\text { Acteurs financés par les } \\
\text { éco-organismes }\end{array}$ & $\begin{array}{l}\text { Financement uniquement des } \\
\text { collectivités locales. }\end{array}$ & $\begin{array}{l}\text { Financement des collectivités locales mais aussi } \\
\text { des distributeurs et des structures de l'économie } \\
\text { sociale et solidaire (ESS). }\end{array}$ \\
\hline $\begin{array}{l}\text { Eco-contribution : ce que } \\
\text { le consommateur voit ou } \\
\text { ne voit pas }\end{array}$ & $\begin{array}{l}\text { Invisible, sauf pour la catégorie Gros } \\
\text { Electroménager Froid. }\end{array}$ & $\begin{array}{l}\text { Visible initialement jusqu'au } 13 \text { février } 2013 \\
\text { (pour financer la collecte des EEE historiques }{ }^{11} \\
\text { ou orphelins }{ }^{12} \text { ), mais prolongée jusqu'en } 2020^{13} \text {. }\end{array}$ \\
\hline $\begin{array}{l}\text { Logique de coordination } \\
\text { entre éco-organismes }\end{array}$ & $\begin{array}{l}\text { Fort rôle de coordination des éco- } \\
\text { organismes qui garantissent les règles } \\
\text { de concurrence. }\end{array}$ & $\begin{array}{l}\text { Faible rôle par rapport au travail des éco- } \\
\text { organismes : missions peu efficaces d'interface } \\
\text { entre collectivités et éco-organismes, et entre ces } \\
\text { derniers pour assurer la régulation. }\end{array}$ \\
\hline $\begin{array}{l}\text { Rôle de l'organisme } \\
\text { coordinateur par rapport } \\
\text { aux autres acteurs. }\end{array}$ & $\begin{array}{l}\text { Le CDC ne conclut des conventions } \\
\text { qu'avec les communes (facultatif pour } \\
\text { elles }^{14} \text { ) et les opérateurs de } \\
\text { traitement }^{15} \text {. }\end{array}$ & $\begin{array}{l}\text { L'OCAD3E est l'interface entre collectivités et } \\
\text { éco-organismes. }\end{array}$ \\
\hline $\begin{array}{l}\text { Importance de la reprise } \\
« 1 \text { pour } 1 »\end{array}$ & $\begin{array}{l}\text { Peu utilisée du fait des contraintes } \\
\text { bureaucratiques (registre des } \\
\text { producteurs }{ }^{16} \text {, procédures à respecter, } \\
\text { etc.) et techniques (règles de stockage, } \\
\ldots)^{17} \text {, et du faible taux de } \\
\text { remboursement par le CDC. }\end{array}$ & $\begin{array}{l}\text { Bien mise en œuvre car un des } 4 \text { éco-organismes } \\
\text { représente les distributeurs. }\end{array}$ \\
\hline
\end{tabular}

\subsection{Les flux au sein du mécanisme « Clearing House » en Italie et en France}

Avant d'entrer dans l'analyse du réseau de gestion des DEEE à l'échelle urbaine, il faut rappeler quelques chiffres concernant la collecte par les éco-organismes mais aussi sur les canaux alternatifs par lesquels les DEEE peuvent finir. Ainsi, si le système de gestion de DEEE dans le cadre du mécanisme « clearing house » est considéré comme efficient, efficace et équitable, il ne faut pas omettre de présenter ses faiblesses pour comprendre pourquoi si peu de déchets empruntent cette voie.

Tout d'abord, l'objectif européen de collecte de $4 \mathrm{~kg} / \mathrm{hab}$./an doit être réalisé à l'échelle nationale, ce qui cache une forte hétérogénéité en termes de performances par les différentes collectivités locales. Ce chiffre peut être considéré comme une cible importante pour les deux systèmes français et italiens. Pourtant, un effort considérable devra être fourni par rapport aux nouveaux objectifs de collecte de $10-12 \mathrm{~kg} / \mathrm{hab}$. en $2018^{18}$ imposés par la révision de la Directive Européenne. Etant donné que le taux de collecte nationale est

\footnotetext{
${ }^{10}$ D'une manière générale en Europe, les situations sont très différentes : d'un seul éco-organisme en Belgique (RECUPEL) jusqu'à une quarantaine au Royaume-Uni.

${ }^{11}$ Qui ont été mis sur le marché avant le 13 aout 2005.

${ }^{12}$ Il s'agit des équipements dont le fabricant ou l'importateur a fait faillite ou a disparu. Voir http://www.europarl.europa.eu/highlights/fr/706.html.

${ }^{13}$ Voir http://www.actu-environnement.com/ae/news/DEEE-eco-participation-repercussion-prorogation-2020-loi-18386.php4.

${ }^{14}$ En absence d'un accord avec les éco-organismes, les municipalités ont la possibilité de gérer les D3E ménagers d'une façon alternative, par exemple en les revendant aux acteurs individuels du traitement.

${ }^{15}$ Même si il existe des exceptions, peu de conventions sont signés avec l'ESS notamment.

${ }^{16}$ Le registre des producteurs doit être rempli par les producteurs ou par les éco-organismes en cas d'organisation collective. Il s'agit d'une obligation règlementaire qui permet d'évaluer la performance des filières par rapport aux objectifs de la directive. Les producteurs et éco-organismes indiquent les données de mises sur le marché des équipements électriques et électroniques, de collecte et de traitement des déchets.

${ }^{17}$ Le Droit italien est en train de produire des actes « in simplificata » pour simplifier les taches des revendeurs

${ }^{18}$ Rapport Ecodom, 2012.
} 
aujourd'hui de l'ordre d'un peu plus de 4kg/hab./an en Italie et de presque $7 \mathrm{~kg} / \mathrm{hab}$./an en France (2012), une telle augmentation de l'efficacité du système représente un défi national important.

Afin de mieux comprendre les deux contextes nationaux, il faut ajouter que la cible atteinte en Italie, c'est-àdire $4 \mathrm{~kg} / \mathrm{hab}$./année, représente environ 260.000 tonnes des déchets électroniques interceptés $(2011)^{19}$, alors que les produits mis sur le marché sont estimés ${ }^{20}$ entre 900.000 et 1.200 .000 tonnes en $2011^{21}$. En France, le taux de $6,9 \mathrm{~kg} / \mathrm{hab}$./an de DEEE collectés représente environ 450.000 tonnes, alors que la mise sur le marché est estimée à environ 1.600.000 tonnes. Ainsi, on voit que le rapport entre les DEEE récupérés par les systèmes des éco-organismes et les produits Hi-Tech mis sur le marché est plutôt faible, de l'ordre de $20 \%$ en Italie et de $30 \%$ en France.

Tableau 2 : Données sur la filière des DEEE en Italie/France et à Milan/Toulouse en 2011

\begin{tabular}{|c|c|c|c|c|}
\hline & Italie & France & Milan & Toulouse \\
\hline $\begin{array}{c}\text { EEE mis sur le } \\
\text { marché (PoM) }\end{array}$ & $\begin{array}{c}\text { Entre } 900.000 \text { et } \\
1.200 .000 \text { tonnes }^{22}\end{array}$ & 1.660 .000 tonnes $^{23}$ & Non connu & Non connu \\
\hline $\begin{array}{c}\text { Kg/hab./année EEE } \\
\text { mis sur le marché }\end{array}$ & $18-20$ & 2524 & Non connu & Non connu \\
\hline DEEE collectés & 260.090 .413 tonnes & 450.000 .000 tonnes $^{25}$ & 3.694 tonnes & 613 tonnes ${ }^{26}$ \\
\hline $\begin{array}{c}\text { Kg/hab./année } \\
\text { DEEE collectés }\end{array}$ & 4,1 & 6,9 & 3,15 & Moins de 1 \\
\hline $\begin{array}{c}\text { PoM/DEEE } \\
\text { collectés }(\%)\end{array}$ & $20 \%$ & $27 \%$ & Non connu & Non connu \\
\hline
\end{tabular}

Ces informations montrent par ailleurs que le système des éco-organismes dans les deux pays n'intercepte qu'une faible part des DEEE générés. Par conséquent, il y a encore une grande quantité de déchets qui, d'une manière plus ou moins connue, emprunte d'autres voies de traitement. Ces flux peuvent suivre les trajectoires suivantes :

- Jetés dans le bac des ordures ménagères par les consommateurs. Dans ce cas, les DEEE sont soit incinérés, soit finissent à la décharge avec les autres déchets.

- Stockés chez les ménages. Ce stockage domestique est très difficile à estimer car il est fréquent, qu'après la phase d'utilisation, les ménages gardent chez eux les équipements usagés pendant un certain temps.

- Repris par le troisième secteur, c'est-à-dire le secteur caritatif et l'achat de seconde main. Dans le cas italien, cela concerne environ 13\% des DEEE générés, tandis qu'en France il est incorporé dans le calcul du taux de collecte du système OCAD3E lorsque les entreprises d'insertion ont contractualisées avec un éco-organisme, ce qui n'est pas toujours le cas. L'ESS est devenu un acteur très important du dispositif car ses structures sont quasiment les seules, avec les activités de revente d'occasion, qui permettent d'exploiter une seconde vie plutôt que de les recycler.

- Récupérés par une nébuleuse d'opérateurs hétérogènes du secteur des déchets (citoyens privés, gens du voyage, petites entreprises qui agissent dans le but de faire des profits en démantelant les DEEE pour revendre les métaux ferreux et non ferreux). Il est ainsi important de rajouter qu'en raison de l'augmentation du prix des métaux depuis les deux dernières années, le marché des matières 'premières secondaires' - récupérables notamment dans les DEEE - est devenu très profitable pour les opérateurs du marché, mais aussi pour les opérateurs du traitement illégal (qui représentent notamment l'antichambre des expéditions à l'étranger)

\footnotetext{
${ }^{19}$ Rapport CDC- Raee, 2012.

${ }^{20}$ Rapport Remedia, 2012.

${ }^{21}$ Ibid.

${ }^{22}$ Rapport Remedia, 2012.

${ }^{23}$ ADEME, 2011.

${ }^{24}$ Ibid.

${ }^{25}$ Ibid.

${ }^{26}$ Cette donnée ne concerne que la Communauté Urbaine Toulouse Métropole, d'après Rapport annuel de la CUTM, 2012.
} 
Cette description générale permet d'introduire les cas d'étude de la gestion de DEEE à Milan et Toulouse. Se concentrer sur ces métropoles fournira d'une part des éléments concernant l'application et la territorialisation de la Directive DEEE dans un contexte local, et permettra d'autre part de conduire une réflexion plus théorique sur la filière des DEEE et d'interroger ce réseau composé par différents cercles pas toujours faciles à mettre en relation.

\section{Grille d'analyse des régimes sociotechniques}

\subsection{Le niveau macro : Le paysage socioéconomique des DEEE}

Le contexte macroéconomique de l'apparition du problème des DEEE est celui d'une société globalisée où règne la consommation de masse. Cela apporte des EEE bon marché et favorise le trafic international de DEEE, alimenté par de fortes inégalités Nord/Sud. Contrairement aux périodes précédant le $20^{\mathrm{e}}$ siècle, le gaspillage s'est depuis largement répandu, soutenu par des modes de conception n'ayant pas pour priorité d'allonger la durée de vie des produits et de les éco-concevoir. Et si la crise a pu réactiver des réflexes d'économie et susciter des mouvements de réutilisation ou de $\mathrm{C} 2 \mathrm{C}$ (consumer-to-consumer) portés par internet, ceux-ci restent marginaux face à l'ampleur de la consommation de masse. Au niveau macropolitique la gestion des DEEE est fortement impactée par les politiques européennes comme la directive DEEE, qui a forcé la mise en place de systèmes formels de gestion des DEEE. Celles-ci ont eu des effets bien au-delà de l'UE car nos importateurs doivent s'y conformer. En termes géopolitiques, l'équilibre des puissances bascule lentement vers l'Asie, où la Chine a pu utiliser son quasi-monopole de production de terres rares à des fins géopolitiques : récupérer ces métaux dans les DEEE européens pourrait alors constituer un réel levier de réduction de notre dépendance à ces matériaux critiques ${ }^{27}$. Enfin, les écosystèmes naturels, qui font partie intégrante du paysage sociotechnique, montrent une résilience de plus en plus faible, que ce soit pour absorber nos déchets ou pour fournir des matières premières. Cela constitue une pression supplémentaire pour changer le régime dominant de gestion des DEEE, qui ne parvient pas à en ralentir la croissance.

\subsection{Le niveau meso : Le réseau d'acteurs à l'échelle urbaine, quelle coordination dans la filière DEEE ?}

La filière de gestion des DEEE s'organise avec des acteurs de sphères hétérogènes qui interviennent à des échelles différentes. Dans les agglomérations milanaise et toulousaine, certains acteurs sont directement présents et localisés sur l'aire urbaine. Ainsi, la filière de gestion des DEEE, pour être efficace, a besoin de coordination entre ces différents acteurs urbains :

- Les collectivités territoriales

- Les distributeurs

- L'économie sociale et solidaire

- Les consommateurs

- les entreprises de collecte et traitement

Il s'agit ainsi de décrire selon les deux métropoles d'étude le positionnement des acteurs et leur performance, ainsi que les interactions entre eux. Si chaque acteur a un rôle bien défini dans la règlementation, il opère différemment dans le réseau selon ses stratégies et ambitions, ce qui influe aussi sur leurs relations avec les autres acteurs. La figure du réseau est donc dépendante de ces nœuds d'interactions.

\subsubsection{Le cas toulousain : Proximité géographique et désengagement des collectivités}

Les deux grandes intercommunalités de l'aire urbaine toulousaine ont délégué leur compétence de traitement des déchets ménagers (deux incinérateurs) à deux grands groupes privés. Avec la mise en place de la réglementation liée aux DEEE en 2007, ces deux intercommunalités informent les habitants, via leur site internet, qu'ils collectent en porte-à-porte les DEEE avec les encombrants. Les habitants pourraient considérer qu'il s'agit d'une collecte sélective des DEEE afin de les orienter vers un recyclage spécifique.

\footnotetext{
${ }^{27}$ Voir l'article d'EcoInfo "Des matériaux critiques pour l'Union Européenne", http://ecoinfo.cnrs.fr/article197.html.
} 
Cependant, ces tournées de collecte des encombrants aboutissent aux installations d'incinération, car les deux entreprises ne considèrent pas que le tri entre DEEE et encombrants soit dans leurs missions initiales puisqu'il nécessite des aménagements spécifiques. Comme cela n'est pas indiqué dans leur contrat de gestion déléguée, les deux entreprises refusent de trier ces déchets et de les orienter vers leur recyclage, malgré la volonté des intercommunalités et l'obligation réglementaire. Si des négociations devraient aboutir pour que ces entreprises changent de position, ce type de situation conduit à des incohérences préjudiciables et montre le manque de contrôle des collectivités urbaines dans cette gestion des DEEE. Les distributeurs ont l'obligation de reprise « 1 pour 1 », mais développent plusieurs stratégies économiques selon l'offre des écoorganismes, ce qui n'est pas sans incidence sur la performance environnementale. Ils sont rémunérés pour cet effort de collecte - au même titre que les collectivités - par les éco-organismes, ce qui est affirmé par ces derniers : «Le secteur de la distribution se place comme les collectivités : une offre de service de DEEE collectés via la signature d'une convention de partenariat assortie d'un soutien financier ${ }^{28}$. Cette rémunération est assez étonnante puisqu'ils sont réglementairement obligés de reprendre le matériel usagé en échange de l'achat d'un neuf. De fait «l'effort écologique des magasins repose sur un service intéressé, ces derniers étant rémunérés en fonction des volumes récupérés » (Bouder et al., 2007). Le choix des magasins sera orienté entre un effort de «masse critique » ou un effort de «massification». La première possibilité réside dans l'approche d'ECO-SYSTEMES, qui rémunère les distributeurs dès lors qu'ils captent un gisement suffisant (mais peu important). Le magasin est donc rétribué pour un faible effort individualiste mais assez critique pour donner lieu à une collecte d'un prestataire logistique choisi par l'éco-organisme. Cette «masse critique » de déchets permet au distributeur de déclencher un enlèvement des lots, d'où une rémunération. Le second scénario tend à utiliser le circuit « aller» de la distribution, c'est-à-dire les centrales d'achat. Le passage par les centrales d'achat permettrait de récupérer les petits lots de chaque magasin afin de massifier un flux important de DEEE. Cette vision est celle d'ERP, qui ne rémunère les distributeurs qu'à partir d'une quantité importante de DEEE collectés. Étant donné qu'il y a un réseau important de distributeurs dans l'agglomération toulousaine, l'effort de collecte est très important via la reprise «1 pour $1 »$. Cela représente 2.538 tonnes, c'est-à-dire $3.7 \mathrm{~kg} / \mathrm{hab}$. selon nos estimations ${ }^{29}$.

Le manque d'implication du consommateur dans le dispositif est une véritable lacune de la filière, car il n'y contribue qu'au titre de l'éco-participation. Il est en effet sollicité financièrement en amont du processus de la gestion des produits en fin de vie (rappelons qu'il y participe aussi financièrement en versant à la collectivité sa contribution pour la gestion des déchets ménagers) :

«La REP se conjugue donc avec une responsabilité élargie des collectivités locales (RECL) et une responsabilité élargie des consommateurs (REC). Un effort «physique » supplémentaire est demandé à ces derniers qui, de plus, subissent en retour les incidences financières de la REP, d'une part en tant que consommateurs, d'autre part en tant que contribuables ; au final, ils paient en tous cas les coûts de post-consommation et leur renchérissement au nom d'une protection accrue de l'environnement. » (Bertolini, 2006, p. 144)

On retrouve dans l'agglomération toulousaine un taux de collecte assez faible $(5.8 \mathrm{~kg} / \mathrm{hab}$.) par rapport à la moyenne nationale (cf. ci-dessus), ce qui correspond à un retard accumulé par les collectivités bien que les distributeurs et les structures de l'économie sociale et solidaire y soient très nombreux. Ainsi, la communauté urbaine de Toulouse Métropole n'a collecté que 613 tonnes $^{30}$ de DEEE en 2011, (cela représente moins de $\left.1 \mathrm{~kg} / \mathrm{hab}^{31}\right)$. Ne fournissant que peu d'effort dans cette filière, la stratégie urbaine consiste à se désengager de la collecte au profit des distributeurs, dont la collecte doit être « une priorité pour l'acheteur d'un $\mathrm{EEE} \gg{ }^{32}$. De plus, le vandalisme dans les déchetteries, les performances habituellement faibles de tri des déchets dans l'habitat vertical et le manque de cohérence entre les structures intercommunales peuvent être avancés comme explications de ces mauvaises performances. Pourtant, de nombreuses entreprises de collecte et prétraitement de DEEE opèrent dans l'agglomération toulousaine et regroupent la majeure partie des flux

\footnotetext{
${ }^{28}$ Entretien avec le Responsable régional d'un éco-organisme, 01/06/09.

${ }^{29}$ Il s'agit d'un chiffre estimé par rapport aux informations du registre des producteurs à l'échelle départementale, des données chiffrées dans le rapport annuel des déchets de Toulouse Métropole, et de la présence importante des structures de l'économie sociale et solidaire dans l'agglomération toulousaine.

${ }^{30}$ Rapport annuel sur le prix et la qualité du service public des déchets de Toulouse Métropole, 2012.

${ }^{31} \mathrm{Il}$ est à noter que ce ratio n'a pas de lien avec les équipements vendus sur le territoire, mais seulement le nombre d'habitants dans la communauté urbaine de Toulouse (714 000 habitants).

${ }^{32}$ Entretien avec responsable - service valorisation élimination Direction Déchets et Moyens Techniques, 2013.
} 
de Midi-Pyrénées (Bahers, 2012). Les tournées de collecte des points d'apport sont une activité logistique lourde et sont limitées si elles sont proches des lieux de consommation. La mobilisation de ce réseau d'entreprises fonctionne grâce à une proximité géographique et organisationnelle (Pecqueur, Zimmerman, 2009 ; Bazin et al. 2009). Cette proximité toulousaine profite d'un bassin d'emploi important et de contraintes logistiques des filières de collecte pour être particulièrement efficace.

L'économie sociale et solidaire risque de se voir détourner de ses idéaux premiers. Son rôle dans la filière et vis-à-vis des éco-organismes est ambigu : les entreprises d'insertion semblent être de plus en plus des cautions sociales d'une filière qui cherche un assentiment du public. Elles sont cantonnées à un travail sur des chaînes de démontage et de manutention logistique. C'est le cas des deux entreprises d'insertion toulousaines retenues par les éco-organismes dans leurs appels d'offre. De plus, ces opérations basiques font face à la concurrence d'entreprises privées, qui seront progressivement privilégiées dans une perspective d'automatisation des procédés et de réduction des coûts. Ainsi, de nombreuses associations d'insertion n'accèdent pas au gisement des éco-organismes pour ces raisons (six dans la région toulousaine). Il s'agit là d'un écueil à éviter qui éloignerait les entreprises d'insertion de l'objectif premier d'insertion professionnelle, pour entrer dans une logique de concurrence sur le plan économique et sur le plan de l'emploi : «Cette économie devient un secteur de sous-traitance de l'économie marchande et participe de la consolidation d'un marché secondaire du travail » (Laville, 2010). Cette utilisation de l'économie sociale et solidaire est regrettable, même si elle participe de 4 l'exemplarité solidaire ${ }^{33}$ de la filière des DEEE. En conclusion, le réseau urbain toulousain ne présente pas des intérêts tous alignés. Bien que tous les acteurs soient présents et impliqués dans la filière toulousaine, deux logiques antagonistes freinent les efforts de gestion :

- Les collectivités considèrent que les distributeurs doivent être plus responsabilisés pour la collecte des DEEE alors qu'elles disposent d'infrastructures efficaces (notamment le réseau des déchetteries).

- Le troisième secteur connait des difficultés à faire entendre sa position et ses atouts face à une logique de minimisation des coûts des éco-organismes.

\subsubsection{Le cas milanais : Effort de la collectivité urbaine et rôle faible des autres acteurs}

Bien que la ville de Milan ait délégué le service de gestion des déchets à une société de capital mixte (AMSA), elle a contractualisé un accord avec le CDC pour la collecte de DEEE ménagers. Ainsi, elle propose plusieurs points de dépôts aux milanais : six déchetteries, ainsi qu'une déchetterie mobile (CAM Centro Ambientale Mobile) et un service de retrait des encombrants «à l'appel ${ }^{34}$. Cependant le taux de collecte de Milan n'a pas encore atteint le seuil réglementaire imposé par la directive de $4 \mathrm{~kg} / \mathrm{hab} . / \mathrm{an}^{35}$ (en $2012: 3,09 \mathrm{~kg} / \mathrm{hab}^{36}{ }^{36}$, c'est-à-dire 3.694 tonnes).

En Italie, le rôle de la distribution est très faible, car « la législation qui a appliqué la Directive DEEE en Italie, pour ce qui concerne les revendeurs, est contradictoire. Cela a effectivement freiné la grande majorité des détaillants dans l'application de leurs fonctions ». (Conférence AssoRecuperi, juin 2013, Milan). À Milan, il s'agit seulement de quelques tonnes de DEEE collectés ${ }^{37}$. Cette carence est l'un des points les plus problématiques dans le réseau d'acteurs à Milan. Les distributeurs connaissent de grandes difficultés à appliquer le principe «1 pour $1 »$. Deux raisons principales en sont la cause : la première concerne le contexte national, alors que la seconde est spécifique à la ville de Milan.

- La transposition du principe 1 pour 1 en Italie fut très compliqué et a engendré des contextes très difficiles pour les distributeurs (cf. ci-dessus : procédures administratives et pratiques très lourdes).

- La difficile négociation entre la collectivité et distributeurs a provoqué un très lent démarrage de la collecte par ces derniers. De plus, la distribution n'a pas eu l'autorisation de s'appuyer sur les points de collecte municipaux avant 2012, ce qui a freiné leur contribution dans la collecte des DEEE.

De plus, l'éco-contribution a toujours été invisible sauf pour la catégorie des GEF (Gros Électroménager Froid) en Italie, ce qui n'a pas été sans causer des controverses concernant la possibilité pour les distributeurs de déguiser le montant de l'éco-contribution. Cette prise de position, dans un contexte où la distribution ne

\footnotetext{
${ }^{33}$ Pour reprendre un vocable d'Emmaüs notamment.

${ }^{34}$ Soumis à des conditions de volume et poids.

${ }^{35}$ Rapport CDC-Raee Lombardia 2011, 2012.

${ }^{36}$ En 20113,15 kg/hab. (ibid.).

${ }^{37}$ Il s'agit seulement de 4 tonnes des DEEE entre janvier et septembre 2011
} 
perçoit pas de remboursements ${ }^{38}$, est une source d'ambiguïtés et permet de souligner l'avantage de la visibilité.

Les citoyens à Milan ont un large panel de possibilités pour recycler leurs DEEE : service de retrait «à l'appel » pour les encombrants, déchetteries municipales, reprise «1 pour 1 » dans les magasins, et apport volontaire dans les structures de l'économie sociale et solidaire - même si le rôle de ces deux derniers acteurs est faible (cf. ci-dessus). Il existe aussi d'autres moyens de se débarrasser des DEEE de manière plus informelle et moins appropriée, tels que le don à des opérateurs informels, le stockage domestique des appareils usagers, et la poubelle des ordures ménagères. À partir de plusieurs enquêtes (AIRES, 2011 ; Remedia - GFK Eurisko, 2011), il est montré que les citoyens ne connaissent pas bien l'organisation de la filière des DEEE. En revanche, lorsqu'ils sont «coutumiers » des déchetteries, ils utilisent ce service public. Ainsi, la collecte à Milan se réalise principalement grâce à la relation efficace entre AMSA $^{39}$ (société délégataire en charge de la gestion des déchetteries) et les Milanais. Les consommateurs sont guidés par l'achat d'un produit et pas forcément intéressés et/ou informés des raisons d'une bonne gestion des déchets générés. Par ailleurs, la littérature sur le comportement de tri (Jackson, 2005) met en évidence que même s'ils sont intéressés et informés, ces facteurs ne sont pas suffisants si les consommateurs ne sont pas à proximité de points de collecte en lien avec leurs espaces quotidiens. Le geste de tri des citoyens est soumis à des conditions de gestion de leur temps («time squeezing » selon Southerton et al., 2003) et de facilité d'accès aux déchetteries ${ }^{40}$. Ces éléments sont des conditions sine qua non pour que l'offre de service rencontre la disponibilité des citoyens. Il faut ajouter que la plupart des déchetteries et points de collecte sont fortement soumis à un système de "dépendance de trajectoires ${ }^{41}$, dans les milieux urbains où la densité immobilière ne permet pas une grande flexibilité d'emplacement, à moins de repenser la localisation des points de collecte ex novo.

Ainsi, le réseau urbain milanais présente des dysfonctionnements et des nœuds faibles dans son articulation. D'abord, il existe un problème normatif important lié à la difficulté d'harmonisation des lois intérieures avec les directives européennes. Ensuite, deux acteurs sont clairement en difficulté dans cette filière :

- Les distributeurs, qui en dehors des contraintes de la loi n'ont aucun intérêt à réaliser la reprise 1 pour 1, car elle représente une charge trop importante avec des aménités très faibles en termes d'image et d'incitations financières. De plus, les distributeurs se sentent floués car leurs contraintes sont beaucoup plus importantes que pour les collectivités.

- L'absence relative du troisième secteur offre peu d'opportunités aux citoyens de donner à des acteurs de proximité des appareils pouvant avoir une seconde vie.

En conclusion de cette comparaison à un niveau meso de ces deux villes, on comprend que l'alignement des intérêts des acteurs n'est pas tout à fait optimale et que les déséquilibres découlent d'interactions différentes. A Toulouse, il s'agit des relations ambigües entre collectivités et distributeurs, puis entre les entreprises d'insertion et les éco-organismes. A Milan, les interactions entre le législateur, les éco-organismes et les distributeurs, ainsi que la faible présence du troisième secteur sont les sources d'un démarrage hésitant de la filière.

\subsection{Le niveau micro : Les niches technologiques du secteur des DEEE}

Les opportunités de «chocs », qui accélèrent la mise en place plus efficace d'un système de gestion des DEEE, sont représentées par l'introduction d'innovations au niveau micro des niches techniques. L'objet HiTech est modifiable selon des facteurs très différents: variables d'éco-conception, d'obsolescence programmée des produits (Ecoinfo, 2012), des possibilités de réparation, d'effets de mode dans un contexte de «société de la consommation» (Baumann, 2005), de miniaturisation de la technologie, etc. Cela influe sur les mises sur le marché de produits Hi-Tech, et par conséquent sur les DEEE générés. Il existe de nombreuses technologies pour traiter les DEEE, qui ne constituent pas toutes des innovations radicales. Pour

\footnotetext{
${ }^{38}$ Il est prévu des primes de rendement pour un bon taux de collecte mais elles ne sont pas suffisantes pour couvrir les coûts réels du service.

${ }^{39}$ Azienda Milanese Servizi Ambientali (Entreprise Milanaise pour les Services Environnementaux).

${ }^{40}$ Nous ne parlons pas forcément de facilité d'accès pour une voiture, mais aussi plutôt de la présence de points de collecte dans la ville en cohérence avec les activités et trajets les plus fréquents des citoyens.

${ }^{41}$ Traduit de « path dependency ».
} 
mieux cerner ces dernières, il convient de présenter la chaîne du recyclage, qui consiste en trois grandes étapes :

1) La collecte des DEEE ;

2) Leur tri, démontage, et prétraitement (notamment mécanique);

3) Leur traitement final :

a. Réparation et réutilisation,

b. Réadaptation,

c. Récupération et réutilisation de modules fonctionnels,

d. Récupération et réutilisation de composants,

e. Récupération de matériaux,

f. Production de matières premières,

g. Récupération d'énergie (valorisation des déchets ultimes par incinération).

L'objectif des étapes 2 et 3 est de prendre en charge les composants et fractions dangereux de manière à éviter les impacts environnementaux et à trouver des solutions rentables pour récupérer ces fractions. Le type de méthode utilisé va dépendre des technologies disponibles et utilisables sur le lieu de collecte, des impératifs législatifs locaux, etc. D'après un rapport de StEP (2009), ces technologies sont spécifiques à chaque catégorie d'EEE ; elles sont résumées dans le tableau ci-dessous ${ }^{42}$.

\section{Tableau 3 : Niches technologiques de traitement des DEEE}

\begin{tabular}{|c|c|}
\hline Les technologies de prétraitement & Les technologies de post-traitement ${ }^{43}$ \\
\hline $\begin{array}{l}\text { La réfrigération et la congélation : } \\
\text { - Technologies de dégazage des fluides refroidissants et de } \\
\text { l'huile; } \\
\text { - Pour les métaux ferreux : déchiqueteuses, machines à } \\
\text { fragmenter, séparateurs magnétiques, séparateur par courants de } \\
\text { Foucault ; } \\
\text { - Séparation par induction magnétique ou par vibrations. } \\
\text { Les installations entièrement automatisées coûtent entre } 2 \text { et } 4 \text { M€ } \\
\text { et nécessitent un entre } 3000 \text { et } 6000 \text { tonnes d'entrants par an. }\end{array}$ & $\begin{array}{l}\text { Les circuits imprimés et les petits appareils électroniques : } \\
\text { - Procédés propriétaires en pyrométallurgie, } \\
\text { hydrométallurgie, et électrométallurgie ; } \\
\text { - systèmes d'épuration des effluents gazeux pour les COV, } \\
\text { dioxines, et gaz acides (refroidisseurs adiabatiques, } \\
\text { déchiqueteuses, filtres, décomposition catalytique); } \\
\text { - UMICORE : ISASMELTM (technologie de combustion à } \\
\text { lance immergée : unité d'extraction par électrolyse et } \\
\text { lixiviation combinant hydro et électrométallurgie) }{ }^{44} \text {. }\end{array}$ \\
\hline $\begin{array}{l}\text { Les appareils TIC: } \\
\text { - Séparateurs de tous types : magnétiques, par courants de } \\
\text { Foucault, par induction magnétique, par vibrations, par densité, } \\
\text { utilisant des propriétés diélectriques ou la température de fusion ; } \\
\text { - Fours de fusion intégrés. }\end{array}$ & $\begin{array}{l}\text { Les fractions métalliques contenant des métaux } \\
\text { précieux (surtout } \mathrm{Cu} \text { ): } \\
\text { - Fonderies de cuivre ; } \\
\text { - Complexes intégrés de fonderie et de raffinerie ; } \\
\text { - Hydrométallurgie. } \\
\text { Fractions métalliques ne contenant pas de métaux } \\
\text { précieux (granules de cuivre et laiton et mélanges des deux, } \\
\text { zinc, plomb, étain, autres mélanges) : fonderies }\end{array}$ \\
\hline \multirow[t]{2}{*}{$\begin{array}{l}\text { Les écrans et téléviseurs : } \\
\text { - Séparation semi-automatique des écrans cathodiques } \\
\text { (découpages : à fil chaud, à choc thermique, au laser, au fil/à la } \\
\text { scie de diamant, au jet d'eau); } \\
\text { - Élimination (semi-)automatique du traitement de surface des } \\
\text { faces avant des écrans cathodiques (billage de plastique, } \\
\text { circulation d'eau, lit fluidisé); } \\
\text { - Déchiquetage automatique des écrans cathodiques et } \\
\text { récupération des fractions métalliques ; } \\
\text { - Récupération des matériaux par tri automatisé (les deux types de } \\
\text { verre peuvent être séparés par différentes techniques : séparation } \\
\text { par densité, par calibrage, par UV/lumière visible ou } \\
\text { fluorescence à rayons X }\end{array}$} & $\begin{array}{l}\text { Aluminium : } \\
\text { - Installations de refonte ; } \\
\text { - Fours tournants et basculants ; } \\
\text { - Utilisation d'un mélange de sodium et de chlorure de } \\
\text { potassium pour capter les impuretés et prévenir } \\
\text { l'oxydation; } \\
\text { - Technologies de pointe utilisées pour éviter les émissions } \\
\text { aériennes de poussières, gaz acides, COV, dioxines ; } \\
\text { - Four d'attente en attendant que l'aluminium soit raffiné. }\end{array}$ \\
\hline & $\begin{array}{l}\text { Métaux ferreux } \\
\text { - Déchiquetage, puis séparation magnétique ou méthodes } \\
\text { semi-automatiques pour séparer les fractions }{ }^{45}\end{array}$ \\
\hline
\end{tabular}

\footnotetext{
${ }^{42}$ Traduction française : rapport final du projet ECOPATENTS, disponible sur http://ecopatents.wp.mines-telecom.fr/.

${ }^{43}$ Les opérations ou équipements impliqués dans ces étapes sont souvent des technologies exclusives ou brevetées. On peut les regrouper dans les 3 catégories suivantes :

- Pyrométallurgie (fours à lance immergée, convertisseurs, fours rotatifs, fours électriques à arc, etc.)

- Hydrométallurgie (solutions acides ou basiques très fortes pour dissoudre et précipiter les métaux de manière sélective comme la lixiviation au chlore)

- Électrométallurgie (extraction électrolytique, électroraffinage du cuivre/zinc/etc.)

${ }^{44}$ Les résidus de métaux précieux sont raffinés dans l'unité ad hoc ; Ag, Au, Pt (métaux de haute pureté) : procédés pyro et hydrométallurgiques développés en interne ; $\mathrm{Pb}, \mathrm{Bi}, \mathrm{Sn}, \mathrm{Ni}, \mathrm{In}, \mathrm{Se}, \mathrm{Sb}, \mathrm{As}$ : haut fourneau à plomb.
} 
En ce qui concerne les technologies environnementales radicales, elles sont difficiles à identifier ex ante car leur potentiel de découplage n'est avéré qu'après leur diffusion. On peut toutefois repérer les tendances des technologies prometteuses de traitement des DEEE en étudiant les brevets déposés pour ce faire. Une étude de l'office international des brevets s'en est chargée, elle a été publiée en novembre 2013 («Patent Landscape Report on E-Waste Recycling Technologies », WIPO ${ }^{46}$ ). Elle souligne que la grande majorité des activités de traitement des DEEE est tout d'abord asiatique, puis européenne. La plupart des brevets sont apparus dans la période postérieure aux années 2000, ce qui représente 9.000 familles de brevets associés aux DEEE. Concernant les activités de traitement, l'innovation se regroupe autour des processus de désassemblage et autres méthodes de séparation ainsi que la décontamination. Pour les matériaux les brevets se concentrent sur les métaux non-ferreux (cuivre, nickel...), les plastiques, les métaux ferreux et les matériaux dangereux (arsenic, antimoine, et surtout le plomb), ainsi que les céramiques, les gommes et les terres rares. Entre 2006 et 2010, on constate une croissance de 38\% des brevets déposés pour la récupération des terres rares, de $19 \%$ pour les métaux précieux, et $14 \%$ pour les métaux non-ferreux.

Pour favoriser la transition vers un régime soutenable de gestion des DEEE, le niveau des niches technologiques dispose donc de technologies de rupture à même de faire basculer le régime dominant vers une soutenabilité plus forte.

\section{Conclusion}

L'approche par les régimes sociotechniques nous a permis d'éclairer les enjeux écologiques complexes des filières des DEEE, tant dans leur compréhension que dans leur résolution. Cette configuration particulière de filière résultant des interrelations entre les parties prenantes est étudiée et comparée selon trois dimensions :

- La dimension macro, c'est-à-dire la transposition en France et en Italie de la directive DEEE et de ses objectifs de transition écologique ;

- La dimension meso, qui représente la territorialisation du dispositif réglementaire des DEEE dans les villes de Toulouse et Milan et implique un changement des régimes sociotechniques selon des facteurs législatifs, logistiques, économiques et socio-culturels ;

- La dimension micro, qui renvoie aux contributions potentielles des niches d'innovation.

Il résulte de cette comparaison une mise à jour de ce qui se cache derrière le fonctionnement du modèle « Clearing house », au travers des relations entre les acteurs impliqués dans la filière et quelles stratégies sont à l'œuvre. Il s'agit de visualiser les différentes manières dont les directives communautaires s'appliquent dans des contextes différents. La comparaison entre les cas milanais et toulousain pointe ainsi les similitudes et divergences relatives à la mise en place très récente de cette filière dans les deux pays. Il en résulte un point de vue plus objectif sur ces systèmes, qui nous a permis d'aller au-delà de sa logique propre pour le mettre à l'épreuve et en repérer des dysfonctionnements. En effet, il n'est pas seulement question du «bon » geste de tri des consommateurs ou de la mise à disposition des points de collecte par la collectivité locale. Il s'agit aussi, au sein de ce système multi-échelle et multi-acteurs, de l'alignement des intérêts de tous les acteurs de la filière. Notre étude de cas montre en effet que la Directive DEEE représente un défi important de changement dans les deux contextes locaux, avec des résultats partiellement satisfaisants démontrés par les faibles performances de collecte. Ainsi, à Milan et à Toulouse les acteurs concernés par la gestion des DEEE ne sont pas forcément «alignés », car leurs positionnements sont toujours déséquilibrés par des logiques et des intérêts différents des objectifs de prévention et recyclage de la réglementation. Enfin, la consommation des objets Hi-Tech est en voie d'augmentation continue, ce qui est peu cohérent avec le principe de réduction des déchets des politiques publiques de gestion des DEEE, et suggère que des solutions technologiques ne suffiront pas pour juguler cette hausse et qu'il convient de coupler des mesures de soutien à l'éco-innovation à des mesures socio-institutionnelles d'inflexion des comportements et d'accompagnement de changements systémiques plus profonds.

\footnotetext{
${ }^{45}$ Voir Cui, J. et al., 2003.

${ }^{46}$ Voir http://www.wipo.int/patentscope/en/programs/patent_landscapes/reports/ewaste.html.
} 


\section{Références bibliographiques}

ADEME, 2012. Rapport annuel sur la mise en œuvre de la réglementation sur les Déchets d'Equipements Electriques et Electroniques (DEEE) Données 2011. Collection Repères

AIRES (Associazione Italiana Retailer Elettrodomestici Specializzati), 2011. Cosa pensano/sanno $i$ consumatori sul tema dei RAEE e sulla Legge « 1 contro 1 ». Ufficio Studi AIRES. http://www.airesitalia.it/ufficiostudi.php

Bahers J.-B., 2012. Dynamiques des filières de récupération-recyclage et écologie territoriale : l'exemple du traitement des déchets d'équipements électriques et électroniques en Midi-Pyrénées. Thèse de doctorat en Géographie-Aménagement de l'espace, CIFRE avec Fournier Métaux SAS, Université de Toulouse 2 Le Mirail, LISST-CIEU, dir. M.-C. Jaillet et A. Rouyer.

Bahers J.-B., 2014. Développement territorial et filière de recyclage (à paraître en 2014), in Sociétés Urbaines et Déchets. Eclairages internationaux, sous la dir. de Claudia Cirelli, Bénédicte Florin, Presses Universitaires François-Rabelais, Tours.

Baumann Z., 2005. Vite di scarto. Ed. Laterza, Roma-Bari.

Bazin S., Beckerich C., Delaplace M., 2009. Les déterminants de l'émergence de filières productives de déconstruction/recyclage : patrimoines productifs locaux, proximités organisées et ou proximité géographique ? $6^{\text {ème }}$ journée de la proximité « Le Temps des Débats »14, 15 et 16 octobre 2009, Poitiers.

Bertolini G., 2006. Responsabilité Elargie des Producteurs : Du Principe aux Applications, dans ACR+, Compendium.

Bouder C., Charlier J.-M., Leray Y., Mevel O., 2007. Enjeux et perspectives de la logistique des retours appliquée à la grande distribution: l'exemple des DEEE. $1^{\text {ère }}$ journée de rechercher relations entre industrie et grande distribution alimentaire. Avignon.

Boulanger P.M., 2008. Une Gouvernance du Changement Sociétal : le Transition Management. La Revue Nouvelle 11, 61-73.

Breuil H., Burette D., Flüry-Hérard B., Cueugniet J., Vignolles D., 2008. Rapport TIC et développement durable. CGEDD (Conseil Général de l'Environnement et du Développement Durable) \& CGTI (Conseil Général des Technologies de l'Information)..

Centro di Coordinamento RAEE, 2012. Rapporto annuale 2012. CDCRaee. http://www.cdcraee.it/GetPage.pub_do?id=402882a13ded0faa013dedfc997201f3.

Centro di Coordinamento RAEE, 2012. Dossier sulla gestione dei rifiuti da apparecchiature elettriche et elettroniche in Lombardia. http://www.cdcraee.it/GetPage.pub do?id=402882a13f084d6a013f099518bb0bd7.

Chilosi M., 2013. Analisi critica dell'attuale sistema di gestione dei RAEE e opportunità di cambiamento alla luce della Nuova Direttiva. La nuova Direttiva RAEE : prospettive ed evoluzione del sistema RAEE, Assorecuperi \& Confcommercio, mars, Milano.

Cui, J. et al., 2003, « Mechanical recycling of waste electric and electronic equipment: A review ». Journal of Hazardous Materials, B99, pp. 243-263.

CUTM (Communauté urbaine de Toulouse métropole), 2011. Rapport annuel sur le prix e la qualité du service publique d'élimination des déchets. Toulouse Métropole. http://www.toulousemetropole.fr/documents/10180/18686/Rapport+2011+Dechets06+\%282\%29.pdf/4f6baf4b-53a6-4ea19c79-efbc67b01e40.

Draetta L., Centemeri L., 2007. La politique italienne en matière de DEEE : Instruments nationaux et mise en œuvre des textes européens In Flipo F., Boutet A., Draetta L., Deltour F., Ecologie des infrastructures numériques. Hermès - Lavoisier.

ÉcoInfo, 2012. Impacts écologiques des TIC : Les faces cachées de l'immatérialité. Les Ulis, EDP Sciences.

Flipo F., Boutet A., Draetta L., Deltour F., 2007. Ecologie des infrastructures numériques. Hermès Lavoisier. 
Flipo F., Gossart C., Deltour F., Gourvennec C., Dobré M., Michot M., Berthet L., 2009. Projet Ecotic Rapport final, Technologies numériques et crise environnementale : peut-on croire aux TIC vertes?

Geels F.W., 2002. Technological transitions as evolutionary reconfiguration processes: a multi-level perspective and a case-study. Research Policy 31, 1257-1274.

Geels F.W., Kemp R., 2007. Dynamics in Socio-technical systems : Typology of Change Processes and Contrasting Case-studies. Technology in Society 29, 441-455.

Geels F.W., Schot J., 2007. Typology of Sociotechnical Transition Pathways. Research Policy 36, 399-417.

Gossart C., 2009. De l'exportation des maux écologiques à l'ère du numérique. Mouvements 60, 23-28.

Huisman J., Khuer R., Maurer C., Magalini F., 2007. 2008 Review of Directive 2002/96 on Waste Electrical and Electronic Equipment (WEEE), Final Report. United Nations University, AEA Technology, Gaiker, Regional Environmental Centre for Central and Eastern Europe, Delft University of Technology, for the European Commission, étude No. 7010401/2006/442493/ETU/G4.

Jackson T., 2005. Motivating sustainable consumption. Centre for Environmental Strategy, University of Surrey.

Kemp R., Rotmans J., 2004. Managing the Transition to Sustainable Mobility In Elzen B., Geels F.W., Ken G., System Innovation and the Transition to Sustainability: Theory, Evidence and Policy.Edward Elgar Publishing.

Kuehr R., Williams E., 2003. Computers and the environment - Understanding and managing their impacts. Kluwer Academic Publishers, United Nations University.

Laville J.-L., 2010. Les raisons d'être des associations In Laville J.-L., Chanial P., Eme B., Dacheux É. Latouche S. et Caillé A., Association, démocratie et société civile. La Découverte /M.A.U.S.S./ C.R.I.D.A. ..

Loorbach D., Frantzeskaki N., Thissen W., 2011. A Transition Research Perspective on Governance for Sustainability In Jaeger C.C., Tàbara J.D., Jaeger J., European Research on Sustainable Development. Springer Berlin Heidelberg.

Pecqueur B., Zimmermann J.-B., 2004. Economie de proximités. Hermès, Paris.

Remedia, 2012. Il sistema nazionale di gestione de RAEE. Studio dei flussi e proposte per il raggiungimento dei target Europei. http://identisweee.net/wp-content/uploads/2012/02/RicercaRaeeRemedia.pdf.

Remedia - GFK Eurisko, 2011. Gli Italiani e il riciclo dei piccoli apparecchi elettronici domestici: comportamento dei cittadini, attività di raccolta $e$ potenzialità di riciclo. http://www.remediapervoi.it/it/news-e-iniziative/iniziative/ricerca-remedia-gfk-eurisko

Southerton D., Chappels H., Van Vliet B., 2004. Sustainable consumption: the implications of changing infrastructures of provision. Edward Elgar Ed., UK.

StEP, 2009. Recycling: from E-Waste to Resources. United Nations Environment Program (UNEP) \& United Nation University (UNU).

United Nations University - Institute for Sustainability and Peace, Politecnico di Milano, IPSOS, 2012. Rapporto Ecodom: I RAEE domestici generati in Italia. Ecodom. http://www.reloaderitalia.it/documents/download/documenti_studi/2012/ecodom_report_2012.pdf

Source : Last draft of [Bahers, J.-B., I. Capurso, et al. (2015). "Réseaux et environnement : Regards croisés sur les filières de gestion des Déchets d'Équipement Électriques et Électroniques à Toulouse et à Milan." Flux 2015/1(99): 32-46.] Version finale publiée sur : https://www.cairn.info/revue-flux-2015-1-page-32.htm. 